\title{
Measurement of Velocity Field of Powder Fluid in Laser Fabrication
}

\author{
Xiuping Chen \\ Laser Processing Center, Tianjin Polytechnic University, Tianjin 300160, China \\ E-mail: chenxiuping963@163.com \\ Zhiquan Chen \\ College of Physics Science and technology,Central South University Hunan, 410083, China \\ Xin Wang \\ Middle school of Guanshan, Ningxiang,Hunan 410643, China
}

\begin{abstract}
The digital image processing method based on DPIV technique was developed to measure the gas-powder two-phase flow velocity field in laser fabrication. The powder flow image measurement process is composed of image collecting, image saving, velocity vector field and flow line group construction. Two adjacent instantaneous images of the same powder flow have been collected by CCD camera. Based on interrelated algorithm, the displacement vector field and the velocity vector field have been calculated. The data of the whole velocity field have been fitted to construct a group of flow line. Under the same process parameters as measurement, the velocity distribution is simulated by FLUENT software, and the simulated result agrees with the measured result. Results show that, the measurement method can be used to obtain the powder flow field velocity distribution online and further design the nozzle size.
\end{abstract}

Keywords: DPIV technique, Velocity field, Coaxial feeding

\section{Introduction}

Laser cladding was developed to become an efficient laser manufacturing technique, and it was able to fabricate and repair 3D metal part (Yang,2002,pp. 234-238). In laser manufacturing, high power laser, metal powder and gas are output from coaxial nozzle. The powder flow is a complicate process, velocity field of the two-phase flow (powder and gas) can obviously affect the shape and function of laser cladding casing. Therefore, the velocity field measurement of the powder flow is important. However, the powder particle is too small and moves too fast to be distinguished using direct measurement method. Up to now, the reasonable and quantitative evaluation method is not complete. Digital Particle Image Velocimetry (DPIV) technique is developed to measure the powder flow field in our work. It could provide almost all information of moving particles in flow field conveniently. Comparing with traditional velocity measurement methods, it is a quick and quantitative method. Measurement of concentration field in powder flow is reported in other paper(Yang,2006,pp.993-997). This paper will lay emphasis on the digital image processing method for construction of velocity field in the powder flow.

\section{Measurement of Velocity Field in Powder Flow by DPIV}

DPIV measurement system of powder flow field mainly consists of double pulse $\mathrm{Nd}^{+}$: YAG laser, sheet light, $\mathrm{CCD}$ camera, synchronous monitor, image plate and computer. Double pulse $\mathrm{Nd}^{+}$: YAG laser was used to illuminate the powder fluid field, its parameters are: $\lambda=532 \mathrm{~nm}$, single pulse energy $220 \mathrm{~mJ}$, pulse repeat frequency $10 \mathrm{~Hz}$, pulse duration $6 \mu$ s. Special quartz cylindrical lens are selected to transform $\mathrm{Nd}^{+}$: YAG laser beam into a 2D sheet light, which have adjustable thickness from $1 \mathrm{~mm}$ to $2.5 \mathrm{~mm}$ and a length from $5 \mathrm{~mm}$ to $35 \mathrm{~mm}$. Frame straddle CCD camera with double-exposure function is used to catch two frame images with the exposure time duration of $1 \mu \mathrm{s} \sim 40 \mathrm{~ms}$. The picture of measurement device of powder flow velocity field is shown as Fig.1.

\subsection{Reconstruction of Velocity Field}

Reconstruction of velocity field mainly consists of image collect, image record, image show timely, image processing 
and image correction. The visualized image processing software has been developed with DELPHI package. Cross correlation algorithm (as shown in Fig.2) are used to calculate the displacement $\Delta \mathrm{d}$ of powder particles in two images (Wang,2000,pp.302-306,Wu,2002,pp.594-598), velocity of particles are obtained from $\Delta \mathrm{d} / \Delta \mathrm{t}$ where $\Delta \mathrm{t}$ is the double pulse duration.

\subsection{Pretreatment}

The aim of pretreatment is to distinguish powder region and non-powder region with concentration criterion. Two CCD original images (format: bmp, size: 1004 pixels $\times 1004$ pixels, exposure duration: $\Delta t=1 \mu \mathrm{s}$ ) of the same powder flow field are shown as Fig.3 (a) and (b).

The white region has metal powder and the black region hasn't because of no light reflection. It is not necessary to process the black region, because the calculating time and the wrong distinguishing probability will be increased. So, the black region must be filtered. The concentration of a small area A in the image of powder flow field can be defined as follows:

$c=\frac{n(A, G)}{p x \times p y}$.

Where $n(A, G)$ is the number of the pixel in area A whose grey value $>\mathrm{G}$ (a given value), $p x \times p y$ is the number of all the pixel of A.

If $c>c^{*}$ (a given value), A can be regarded as an area with powder, otherwise, $\mathrm{A}$ is non-powder region. The pretreatment computing program flow can be seen in Fig. 4 .

\subsection{Cross Correlated Coefficient}

Cross correlated coefficient is used to describe the similar characteristic about two small regions. The cross correlated coefficient of two same size grey matrixes is defined as (Sun, 2000, pp.364-367,Wu,2004,pp.623-628):

$R s(m, n)=\frac{\sum_{j} \sum_{i} G_{1}(i, j) G_{2}(i+n, j+m)}{\sqrt{\left(\sum_{j} \sum_{i} G_{1}^{2}(i, j)\right)\left(\sum_{j} \sum_{i} G_{2}^{2}(i+n, j+m)\right)}}$.

Where $G_{k}(x, y)$ is the grey value of the pixel point(x,y)in grey matrix $G_{k}(\mathrm{k}=1,2), \mathrm{m}$ and $\mathrm{n}$ are the differences between $G_{1}$ and $G_{2}$ in $\mathrm{x}$ and y direction respectively, Rs is the cross correlated coefficient of grey matrix $G_{k}(\mathrm{k}=1,2)$. $G_{k}(x, y) \in[0,255], R s(m, n) \in[0,1]$.

\subsection{Velocity Vector Field}

The velocity field can be expressed as:

$$
E_{u v}\left\{\left(x_{a i}, y_{a i}\right), \frac{\Delta x_{i}}{\Delta t}, \frac{\Delta y_{i}}{\Delta t}, t \mid i=0,1,2,3 \ldots\right\} .
$$

Where $E_{u v}$ is the velocity field at moment $\mathrm{t}, \frac{\Delta x_{i}}{\Delta t}$ and $\frac{\Delta y_{i}}{\Delta t}$ are respectively the velocity in $\mathrm{x}$ and $\mathrm{y}$ direction of pixel point $\left(x_{a i}, y_{a i}\right) . \Delta x_{i}=x_{b i} *-x_{a i}, \Delta y_{i}=y_{b i} *-y_{a i}$. Where $\left(x_{b i}^{*}, y_{b i}{ }^{*}\right)$ is the head position of No.i sub-region in Fig.3 (b) which is the most similar to the sub-region in Fig.3 (a) whose head position is $\left(x_{a i}, y_{a i}\right)$. This can be expressed as:

$$
R s\left(x_{b i} *-x_{a i}, y_{b i} *-y_{a i}\right)=\max _{j}\left[R s\left(x_{b j}-x_{a i}, y_{b j}-y_{a i}\right)\right] \text {. }
$$

The velocity vector field flow is shown as Fig.5. When the program runs, velocity values in $\mathrm{x}$ and $\mathrm{y}$ direction are stored in array $u[i, j]$ and $v[i, j]$.

\subsection{Fitting the Velocity Data}

In order to draw velocity vector flow lines, consecutive function can be built up from $u[i, j]$ and $v[i, j]$.

Let: 
$\left[\begin{array}{l}u(i, j) \\ v(i, j)\end{array}\right]=\left[\begin{array}{llll}u_{1} & u_{2} & u_{3} & u_{4} \\ v_{1} & v_{2} & v_{3} & v_{4}\end{array}\right]\left[\begin{array}{c}j i \\ j \\ i \\ 1\end{array}\right]$.

Where $\left[\begin{array}{l}u(i, j) \\ v(i, j)\end{array}\right]$ is the fitting function of $\left[\begin{array}{l}u[i, j] \\ v[i, j]\end{array}\right]$

The fitting error $\delta$ is expressed as:

$\delta=\left[\begin{array}{c}\sum_{j} \sum_{i}(u(i, j)-u[i, j])^{2} \\ \sum_{j} \sum_{i}(v(i, j)-v[i, j])^{2}\end{array}\right]=\left[\begin{array}{c}\delta u \\ \delta v\end{array}\right]$.

In order to get the minimum of $\delta u$ and $\delta v$, let:

$\frac{\partial \delta u}{\partial u_{k}}=\frac{\partial \delta v}{\partial v_{k}}=0$.

Where $k=1,2,3,4$.

Take $u_{k}$ for example:

$A U=C$.

Where:

$$
\begin{aligned}
& A=A^{T}=\left[\begin{array}{llll}
\sum_{j} \sum_{i}(j i)^{2} & \sum_{j} \sum_{i} j i^{2} & \sum_{j} \sum_{i} j^{2} i & \sum_{j} \sum_{i} j i \\
\sum_{j} \sum_{i} j i^{2} & \sum_{j} \sum_{i} i^{2} & \sum_{j} \sum_{i} j i & \sum_{j} \sum_{i} i \\
\sum_{j} \sum_{i} j^{2} i & \sum_{j}^{j} \sum_{i} j i & \sum_{j} \sum_{i} j^{2} & \sum_{j}^{j} \sum_{i} j \\
\sum_{j} \sum_{i} j i & \sum_{j} \sum_{i} i & \sum_{j} \sum_{i} j & \sum_{j}^{j} \sum_{i}^{1} 1
\end{array}\right] . \\
& U^{T}=\left[\begin{array}{llll}
u_{1} & u_{2} & u_{3} & u_{4}
\end{array}\right] . \\
& C^{T}=\left[\begin{array}{llll}
\sum_{j} \sum_{i} u[i, j] j i & \sum_{j} \sum_{i} u[i, j] i & \sum_{j} \sum_{i} u[i, j] j & \sum_{j} \sum_{i} u[i, j]
\end{array}\right] .
\end{aligned}
$$

The coefficients in $u(i, j)$ can be obtained by:

$U=A^{-1} C$.

\subsection{Velocity Vector Flow Line Group}

Velocity vector flow line is to describe the flow direction of a flow field (Tian,2001,pp.211-213,Wei,2001,pp.14-19). At a point in the velocity vector flow line, the directions of tangent and velocity vector (at the same point) are identical. The velocity vector flow line equation can be express as:

$\frac{d x}{u(x, y)}=\frac{d y}{v(x, y)}$.

Where $(\mathrm{x}, \mathrm{y})$ is a point in the flow field.

\section{Measured Result and Calculated Result}

Under the process parameters: initial powder volume fraction at the nozzle entrance $c=0.1$ and initial gas velocity $\mathrm{u}=3 \mathrm{~m} / \mathrm{s}$, the experimental results are obtained from Fig. 6 to Fig. 8 .

As shown in Fig.6, velocity vector field can be obtained in about $375 \mu$ s by this software. The small arrows' lengths stand for velocity magnitudes, and the arrows' directions stand for velocity vector direction. Above the focused region, powder from two sides of nozzle moves face to face. Below the focused region, the powders of two roads leave each other duo to collision. Velocity increases gradually along the gravity direction (the direction of stand-off distance). And 
the velocity of powder far away from the focused region tends to be identical and vertical.

Velocity vector flow line group, and focused region is shown in Fig.7. Above the focused region, the velocity vector flow line meander to the focused region duo to the direction of the powder particles output from the nozzle; below the focused region, the vector flow lines diverge because of collision. The circle region is the focused region, and the center of the circle is the focused point. The focused information is given in "OUTPUT DATA" frame: focused point: $(43.65 \mathrm{~mm}, 8.46 \mathrm{~mm})$, upper focused angle: $9.43^{\circ}$, lower focused angle: $3.19^{\circ}$, focused radium: $3.83 \mathrm{~mm}$.

Comparison between the calculated value and the measured value of the velocity distribution on the nozzle's center line are shown in Fig.8. The calculated value is the simulated result based on FLUENT software under the same process parameters which we get the measured value. The powder velocity magnitude increases along the direction of stand-off distance both in calculated value and measured value. In front of the stand-off distance about $40 \mathrm{~mm}$, the measured value is higher than the calculated value, but behind $40 \mathrm{~mm}$ they agree with each other better. It can be supposed that the two values will agree with each other much better if the measurement step is selected smaller enough.

\section{Summary}

1) The digital image measurement software of velocity field based on DPIV technique has been successfully developed. The velocity vector distribution, velocity vector flow line and information of the focused point can be calculated within about 350 450us. The efficient of metal powder flow field measurement is improved greatly.

2) Under the same process parameters, the velocity distribution is simulated by FLUENT software. The calculated result agrees with the measured result, which indicates that the measurement is reliable.

\section{References}

Sun, Hequan and Kang, Haigui. (2000). Journal of Dalian University of technology, 40(3), 364-367.

Tian, Wendong, Wei, Xiaolin and Sheng, Hongzhi. (2001). Journal of Hydrodynamics, 16(2), 211-213.

Wang, Yanting, Zhang, Yongming and Liao, Guangxuan. (2000). Journal of China University of Science and Technology, 30(3), 302-306.

Wei, Runjie. (2001). Beijing: Beijing University of Aeronautics and Astronautic. pp. 14-19.

Wu. Longhua, Yan, Zhongmin and Tang, hongwu. (2002). Advances in water science. 13(5), 594-598.

Wu. Longhua. (2004). Journal of hydrodynamics, 19(5), 623-628.

Yang. Sen, Zhong. Minlin and Zhang, Qingmao. (2002). Powder metallurgy technology, 20(4), 234-238.

Yang Xichen, Lei Jianbo, Yang Nan. (2006 ). Chinese journal of laser, 33(7), 993-997.

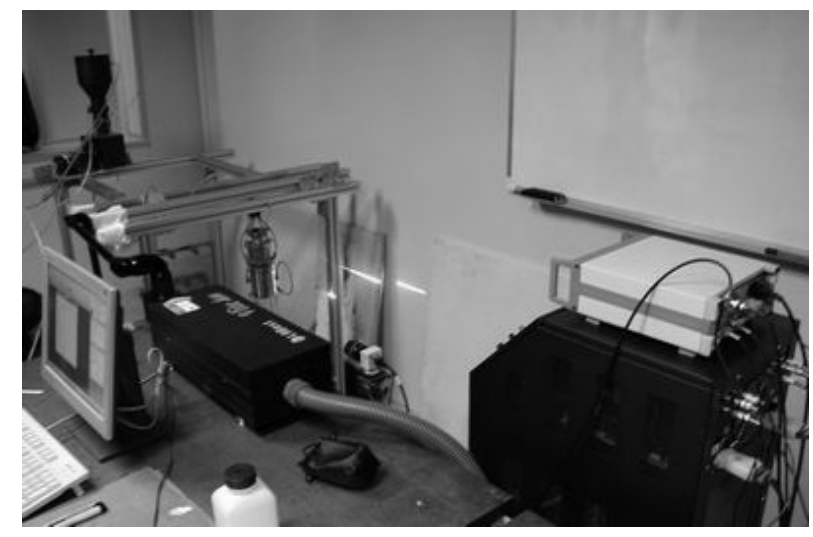

Figure 1. The measurement device of powder flow velocity field 


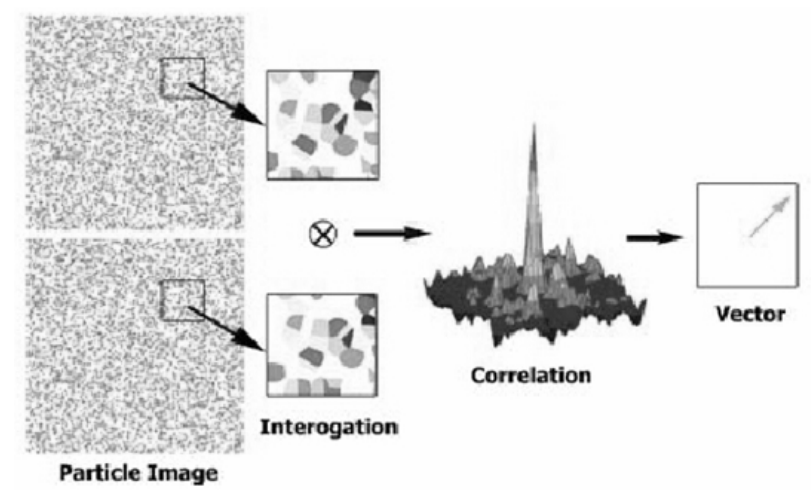

Figure 2. Cross correlation algorithm for calculating velocity of powder particles by DPIV

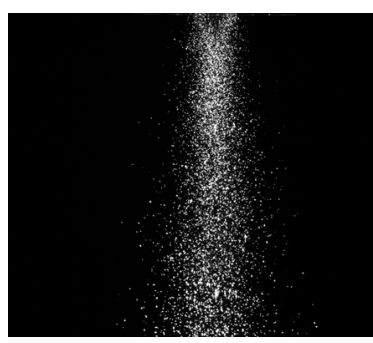

(a)

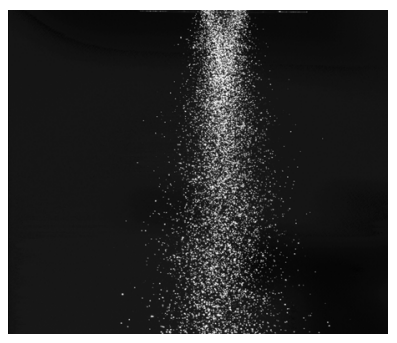

(b)

Figure 3. Powder flow images at moment $\mathrm{t}$ (a) and at moment $\mathrm{t}+\Delta t(\mathrm{~b})$

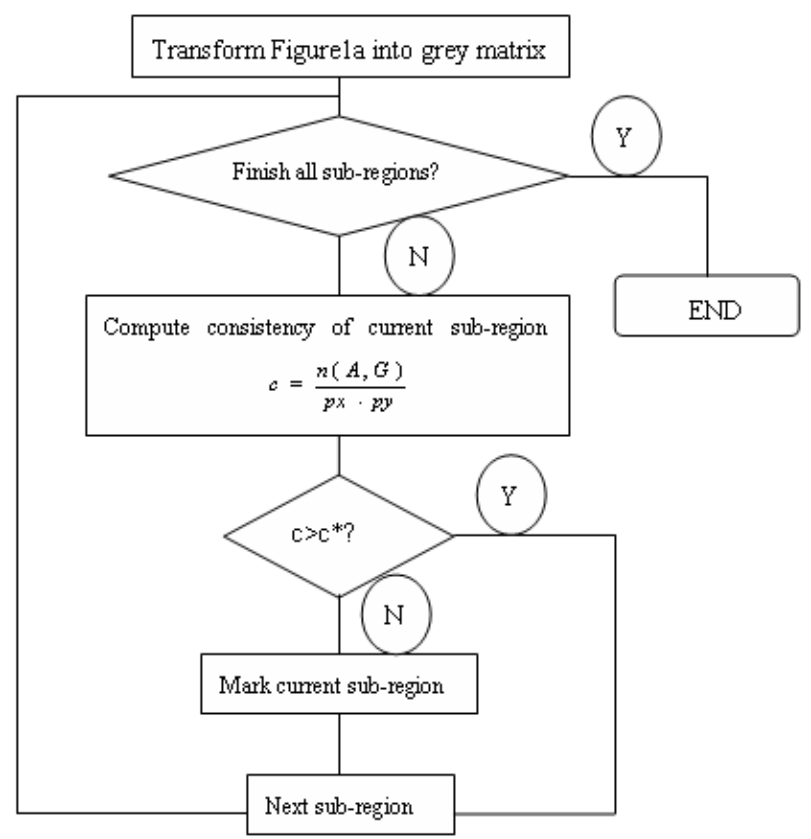

Figure 4. Pretreatment computing program flow 


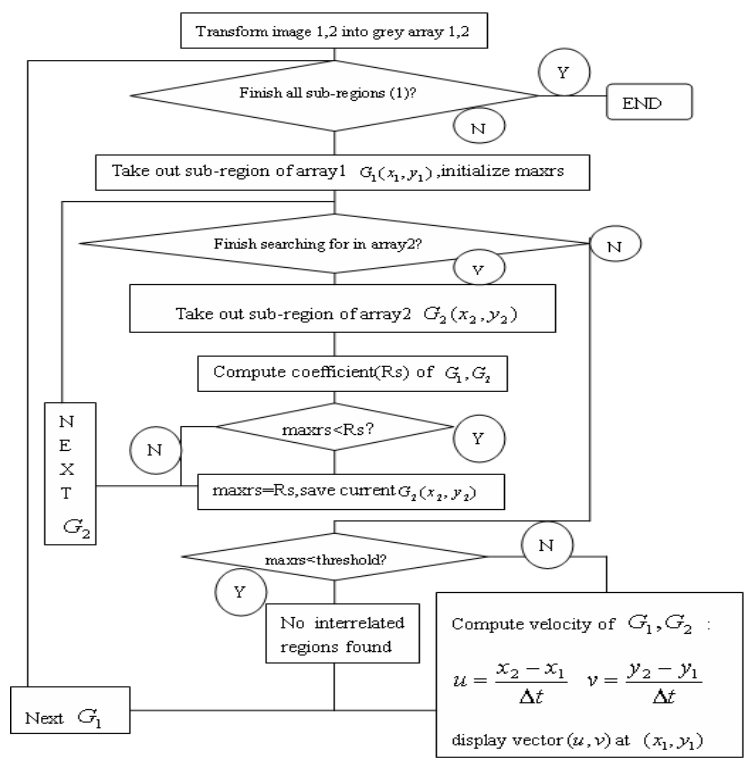

Figure 5. Velocity vector field flow

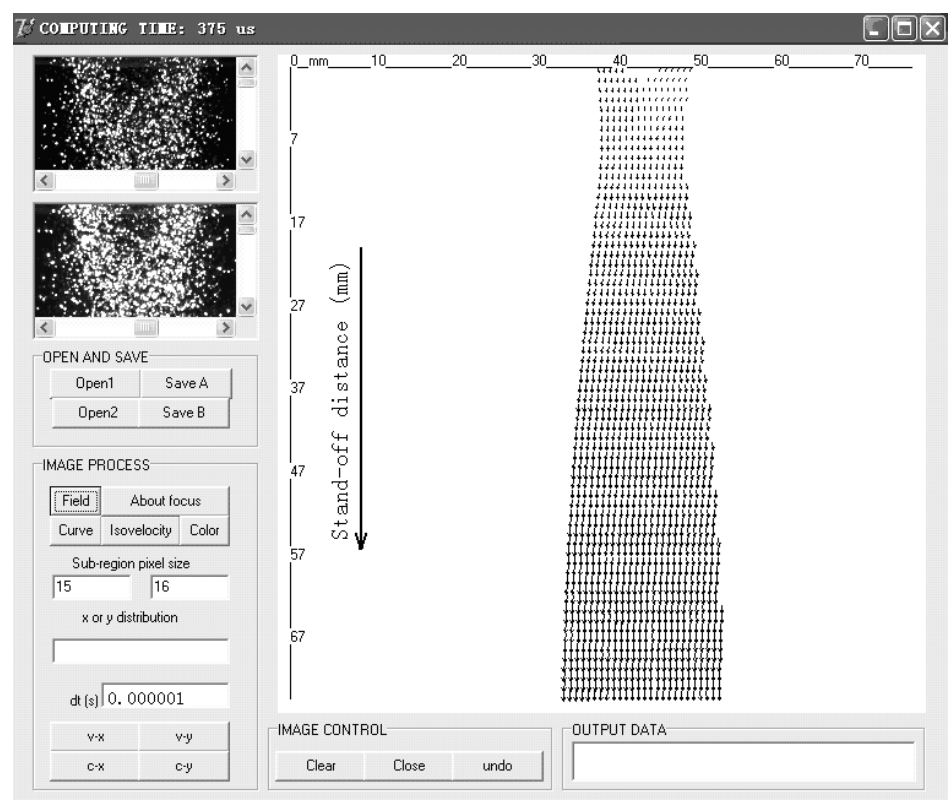

Figure 6.Velocity vector field 


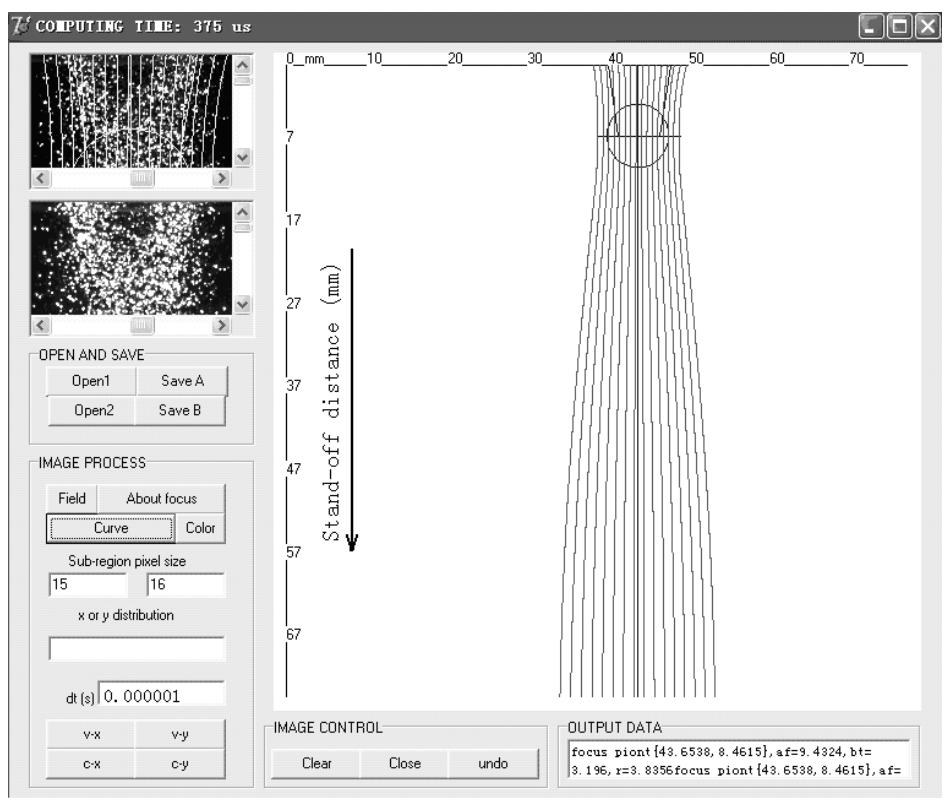

Figure 7. Flow line group and focused region information

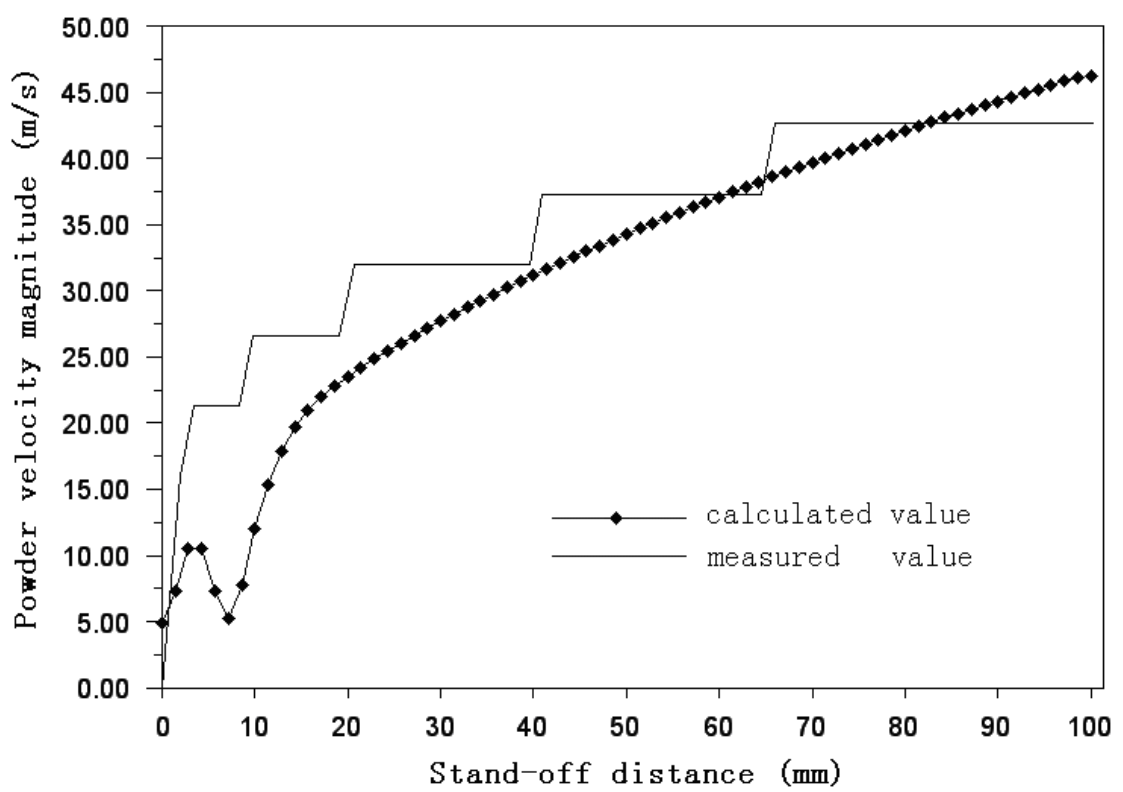

Figure 8. Comparison between calculated value and measured value of the velocity distribution on the nozzle's center line 